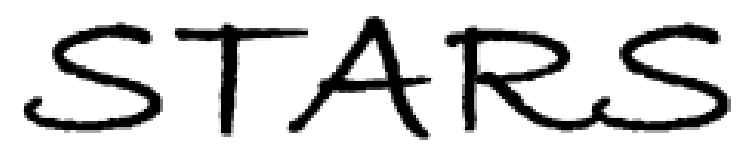

University of Central Florida

STARS

$1-1-2008$

\title{
Reducing the color shift of a multidomain vertical alignment liquid crystal display using dual threshold voltages
}

Ruibo Lu

University of Central Florida

Shin-Tson Wu

University of Central Florida

Seung Hee Lee

Find similar works at: https://stars.library.ucf.edu/facultybib2000

University of Central Florida Libraries http://library.ucf.edu

This Article is brought to you for free and open access by the Faculty Bibliography at STARS. It has been accepted for inclusion in Faculty Bibliography 2000 s by an authorized administrator of STARS. For more information, please contact STARS@ucf.edu.

\section{Recommended Citation}

Lu, Ruibo; Wu, Shin-Tson; and Lee, Seung Hee, "Reducing the color shift of a multidomain vertical alignment liquid crystal display using dual threshold voltages" (2008). Faculty Bibliography 2000s. 650. https://stars.library.ucf.edu/facultybib2000/650

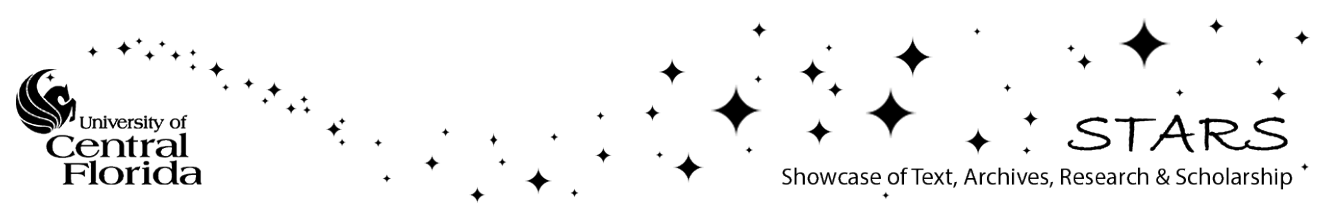




\section{Reducing the color shift of a multidomain vertical alignment liquid crystal display using dual threshold voltages}

Cite as: Appl. Phys. Lett. 92, 051114 (2008); https://doi.org/10.1063/1.2838752

Submitted: 27 November 2007 . Accepted: 08 January 2008. Published Online: 08 February 2008

Ruibo Lu, Shin-Tson Wu, and Seung Hee Lee

\section{ARTICLES YOU MAY BE INTERESTED IN}

Electro-optic characteristics and switching principle of a nematic liquid crystal cell controlled by fringe-field switching

Applied Physics Letters 73, 2881 (1998); https://doi.org/10.1063/1.122617

Low voltage blue-phase liquid crystal displays

Applied Physics Letters 95, 231101 (2009); https://doi.org/10.1063/1.3271771

Electro-optics of polymer-stabilized blue phase liquid crystal displays

Applied Physics Letters 94, 101104 (2009); https://doi.org/10.1063/1.3097355

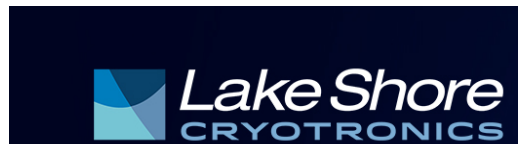

Sensors, Controllers, Monitors

from the world leader in cryogenic thermometry 


\title{
Reducing the color shift of a multidomain vertical alignment liquid crystal display using dual threshold voltages
}

\author{
Ruibo Lu and Shin-Tson $\mathrm{Wu}^{\mathrm{a})}$ \\ College of Optics and Photonics, University of Central Florida, Orlando, Florida 32816, USA \\ Seung Hee Lee \\ School of Advanced Materials Engineering, Chonbuk National University, Chonju, \\ honbuk 561-756, Republic of Korea
}

(Received 27 November 2007; accepted 8 January 2008; published online 8 February 2008)

\begin{abstract}
A multidomain vertical alignment liquid crystal display (MVA LCD) with reduced color shift is proposed. Each pixel is divided into a main region and a subregion. A thin electric shielding layer is embedded in the subregion to generate a higher threshold voltage than that of the main region. As a result, the final gamma curve is a superposition of two different-shaped gamma curves. Such a MVA LCD exhibits a reduced color shift while only requires a single thin-film transistor. Its potential application for LCD TVs is emphasized. () 2008 American Institute of Physics.
\end{abstract}

[DOI: $10.1063 / 1.2838752$ ]

For large-screen liquid crystal displays (LCDs), high contrast ratio, wide viewing angle, and excellent color performance such as small color shift and good angulardependent color uniformity all have to be satisfied simultaneously. ${ }^{1}$ Multidomain vertical alignment (MVA) LCDs exhibit a high contrast ratio and wide viewing angle and have become the mainstream approach for LCD TVs. Currently, four-domain and eight-domain VA liquid crystal (LC) configurations are commonly practiced by the adoption of protrusions or slits on the device substrates. ${ }^{2}$ With the help of compensation films, the viewing angle of a MVA-LCD can reach over $100: 1$ at the $\pm 80^{\circ}$ viewing cone. ${ }^{3}$ However, as compared to the multidomain in-plane switching mode, ${ }^{4,5}$ MVA has inferior performances in color shift and angular color uniformity. Especially, MVA shows an evident gamut curve distortion at the large oblique viewing angles. ${ }^{6}$

Some approaches, such as capacitive coupled method and two thin-film-transistor (TFT) method, have been proposed to improve the gamma curve of a MVA LCD. ${ }^{7,8} \mathrm{Al}-$ though these methods can reduce the off-axis color shift, the required electronic circuit is rather sophisticated. Moreover, using two TFTs would reduce the aperture ratio which, in turn, lowers the optical efficiency and increases the power consumption.

In this paper, we propose a dual threshold voltage method for reducing color shift. In our design, each unit pixel is divided into a main pixel and a subpixel. In the subpixel, a thin electric shielding layer (ESL) is embedded. This ESL shields a portion of the applied voltage so that the threshold voltage is higher than that of the main pixel in which there is no ESL. Besides the reduced color shift, this MVA LCD only requires a single TFT on each unit pixel. As a result, the aperture ratio is high which is important for low power consumption.

Figure 1(a) shows the plane view of the MVA LCD and Fig. 1(b) shows the schematic cross-sectional view along the line $A-A^{\prime}$, as indicated in Fig. 1(a). From Fig. 1(b), the inner surface of the top glass substrate is deposited with red, green, and blue (RGB) color filters, and then covered with a layer

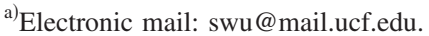

of indium tin oxide (ITO) as common electrode. Each common electrode has a plurality of domain guiding layers, which are the LC alignment slits formed by the opening patterns through the photolithographic etching of the transparent common electrode. On the inner surface of the bottom glass substrate, the ITO electrode is divided into two parts: main region and subregion, as shown in Fig. 1(b). In the main region, ITO is laid on top of an overcoating layer. While in the subregion, a passivation layer such as silicon oxides $\left(\mathrm{SiO}_{x}\right)$ is deposited on top of ITO to work as an electric shielding layer to screen a portion of the applied voltage. As a result, the main region has a lower threshold voltage than the subregion. The unit pixel is driven by a single TFT (not shown).

A liquid crystal layer is vertically aligned between the bottom and top substrates. When the applied voltage exceeds a threshold, the LC directors are tilted into different directions with the aid of the domain guiding layers to form mul-

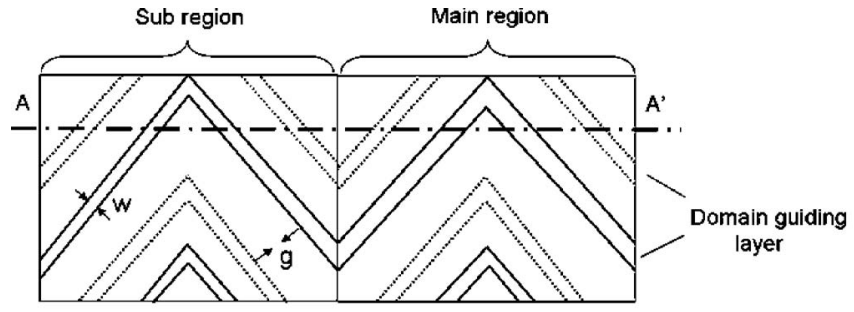

(a)

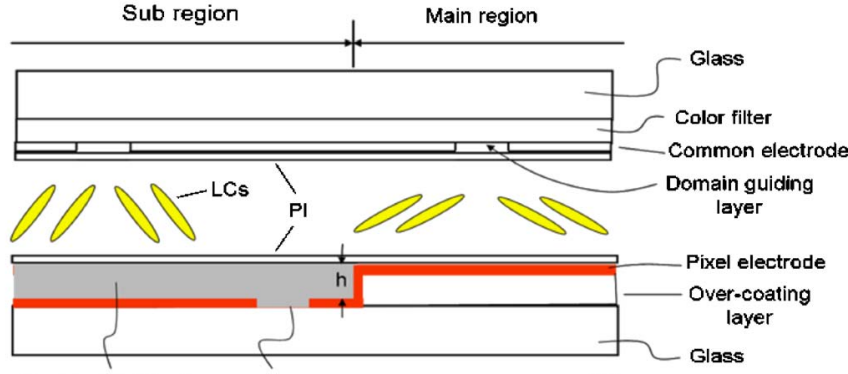

Electric shielding layer Domain guiding layer

(b)

FIG. 1. (Color online) (a) The plane view of the proposed MVA LCD and (b) a schematic cross-sectional view along line $A-A^{\prime}$ in (a). 


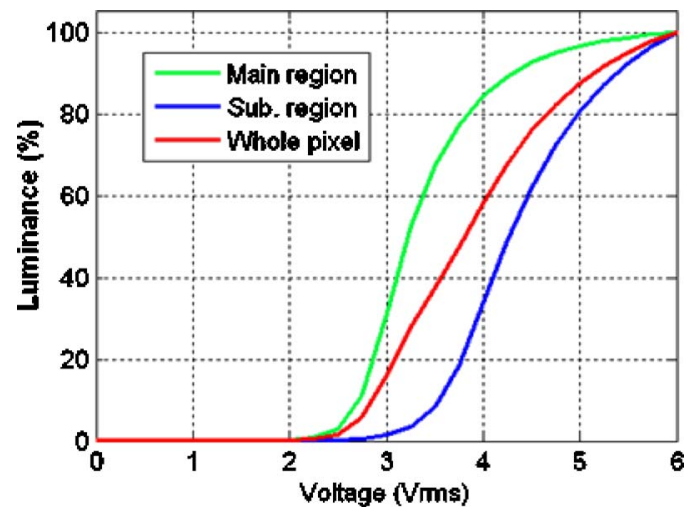

FIG. 2. (Color online) The simulated voltage-dependent luminance curves under the white light source at different regions.

tidomain LC configurations. For the zigzag shaped domain guiding layers, eight domains are formed: four in the main region and another four in the subregion because they have different threshold voltages. A wide view angle can be obtained for the MVA LCD when proper compensation films, such as uniaxial $a$ and $c$ plates are employed. ${ }^{3}$

When a voltage $V$ is applied to the LC cell, the effective voltage across the LC layer $\left(V_{\mathrm{LC}}\right)$ in the subpixel region is reduced to $V_{\mathrm{LC}}=V \times C_{\mathrm{LC}} /\left(C_{\mathrm{LC}}+C_{\mathrm{ESL}}\right)$, where $C_{\mathrm{LC}}$ and $C_{\mathrm{ESL}}$ stand for the capacitance of the LC and ESL, respectively. The magnitude of this voltage shielding depends on the ESL capacitance, which is determined by the area of the ESL and material dielectric constant. For a given dielectric material employed, we can still obtain the desirable threshold voltage difference between the main and subpixels by controlling the ESL thickness. The gamma curve of the whole pixel is a superposition from those of main and subregions. Moreover, the area ratio of the main region and subregion offers another degree of freedom to optimize the final shape of the gamma curve.

Let us take one example to illustrate the design principle and device performance. Here, the area ratio between the main and subregions is varied from 1:1 to $1: 4$. We assume the repeated unit pixel size of the proposed MVA LCD is $100 \mu \mathrm{m}$ (width) $\times 300 \mu \mathrm{m}$ (height) to realize an eightdomain LC configuration corresponding to a conventional four-domain MVA LCD with the same pixel size and design parameters. The slit-type domain guiding layers have zigzag shapes [as shown in Fig. 1(a)] with width $w=12 \mu \mathrm{m}$, and the gap between the neighboring domain guiding layers on the projection plane is $g=35 \mu \mathrm{m}$. The flat ESL is made of $\mathrm{SiO}_{x}$, whose height determines the threshold voltage difference between the main and subpixels. To obtain a desirable threshold voltage difference between the main and subpixels, we chose the $\mathrm{SiO}_{x}$ height to be $h=1.2 \mu \mathrm{m}$. The cell gap between the top and bottom substrates is $d=4 \mu \mathrm{m}$ and a Merck negative $\Delta \varepsilon$ LC mixture MLC-6608 (birefringence $\Delta n=0.083$ at $\lambda=550 \mathrm{~nm}$, dielectric anisotropy $\Delta \varepsilon=-4.2$ and rotational viscosity $\gamma_{1}=0.186 \mathrm{~Pa} \mathrm{~s}$ ) is aligned vertically to the substrates in the initial state. Its azimuthal angle is $0^{\circ}$ and pretilt angle is $90^{\circ}$.

Figure 2 shows the voltage-dependent luminance curves through the main region, the subregion, and the whole unit pixel that consists of both regions. The incident white light source is from a conventional cold cathode fluorescent lamp backlight, passing through the RGB color filters before en- tering the MVA LCD panel which is sandwiched between two crossed linear polarizers. During calculations, the wavelength-dependent birefringence ${ }^{9}$ of the LC material was taken into consideration. The threshold voltage of the main region is $2.25 \mathrm{~V}_{\mathrm{rms}}$ while the subregion is $2.80 \mathrm{~V}_{\mathrm{rms}}$. It can be seen that due to the existence of the ESL, the threshold voltage of the subregion is increased noticeably. Therefore, the subregion has a lower luminance than the main region under the same gray level defined by the whole pixel.

To quantitatively characterize the off-axis image quality, an off-axis image distortion index, $D(\theta, \varphi)$, is defined as ${ }^{7}$
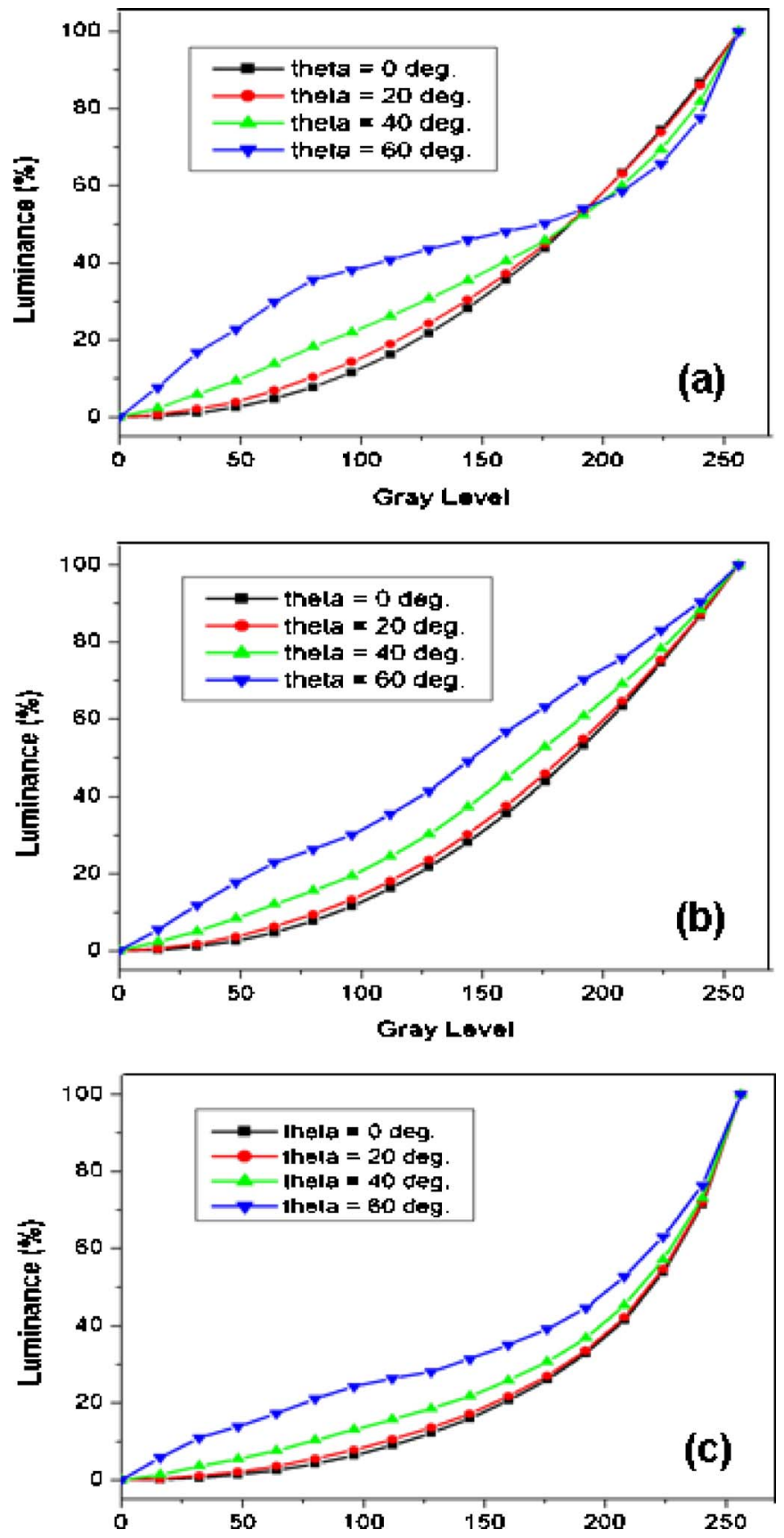

FIG. 3. (Color online) Typical gamma curves at different incident angles with a gamma correction factor $\gamma=2.2$ in (a) the conventional MVA LCD, (b) the proposed MVA LCD with main/subarea ratio of 1:1, and (c) the proposed MVA LCD with main/subarea ratio of 1:4. 


$$
D(\theta, \varphi)=\left\langle\frac{\left|\Delta B_{i, j(\text { on axis })}-\Delta B_{i, j(\text { off axis }, \theta, \varphi)}\right|}{\Delta B_{i, j(\text { on axis })}}\right\rangle_{i, j=0-255} .
$$

Here, $\Delta B_{i, j}$ is the brightness difference between the $i$ th and $j$ th gray levels, and \langle\rangle denotes the average for all cases of arbitrary gray levels. $D(\theta, \varphi)$ is within the range from 0 to 1 . A smaller $D(\theta, \varphi)$ implies to a smaller image distortion as represented from the angular-dependent gamma curves, i.e., a better off-axis image quality.

Figure 3 compares the simulated gamma curves of three different MVA LCDs at different incident angles with a gamma correction factor $\gamma=2.2$. Here, the azimuthal angle is set at $0^{\circ}$ and an 8 bit grayscale with 256 gray levels is evaluated. As calculated from Eq. (1), the conventional fourdomain MVA LCD, as depicted in Fig. 3(a), has a $D$ value of 0.3510 at $(\theta, \varphi)=\left(60^{\circ}, 0^{\circ}\right)$ viewing direction. Figure 3(b) depicts the results for the eight-domain MVA LCD with the main/subarea ratio of $1: 1$. Its $D$ value is 0.2506 at $(\theta, \varphi)$ $=\left(60^{\circ}, 0^{\circ}\right)$ viewing direction which is $28.6 \%$ improved over the conventional four-domain MVA LCD. This indicates that a MVA LCD with dual threshold voltages indeed reduces the color shift at off-axis viewing directions. The $D$ value can be further reduced by optimizing the main/subarea ratios. For example, when the main/subarea ratio is at $1: 4$, the eightdomain MVA LCD has a $D$ value of 0.1629. As Fig. 3(c) shows, the color shift is reduced. However, the effective luminance in the low gray levels is sacrificed because the subregion has a larger area and a higher threshold voltage. Since the proposed MVA LCD only requires a single TFT for the entire unit pixel, its manufacturing cost should be lower and aperture ratio higher than that of the two-TFT approach. Our approach is particularly desirable for LCD TV applications.

In conclusion, we have developed a low color shift MVA LCD using dual threshold voltages for the main and subregions of a unit pixel. The different threshold voltages can be achieved by controlling the thickness of the electric shielding layer in the subregion or the area ratio of the main and subregions. This approach not only reduces the color shift at off axis but also requires only one TFT for each unit pixel which improves the aperture ratio.

The authors are indebted to the financial support from Chi-Mei Optoelectronics, Taiwan.

${ }^{1}$ D. K. Yang and S. T. Wu, Fundamentals of Liquid Crystal Devices (Wiley, New York, 2006).

${ }^{2}$ R. Lu, X. Zhu, S. T. Wu, Q. Hong, and T. X. Wu, J. Disp. Technol. 1, 3 (2005).

${ }^{3}$ Q. Hong, T. X. Wu, X. Zhu, R. Lu, and S. T. Wu, Appl. Phys. Lett. 86, 121107 (2005).

${ }^{4}$ H. Hong, H. Shin, and I. Chung, J. Disp. Technol. 3, 361 (2007).

${ }^{5}$ R. Lu, Q. Hong, Z. Ge, and S. T. Wu, Opt. Express 14, 6243 (2006).

${ }^{6}$ H. C. Jin, I. B. Kang, E. S. Jang, H. M. Moon, C. H. Oh, S. H. Lee, and S. D. Yeo, J. Soc. Inf. Disp. 15, 277 (2007).

${ }^{7}$ S. S. Kim, SID Int. Symp. Digest Tech. Papers 36, 1842 (2005)

${ }^{8}$ C. C. Liu, Y. P. Chang, M. C. Tai, M. H. Chang, and M. T. Wang, Proceedings of the 13th International Display Workshops, 2006 (unpublished), p. 625.

${ }^{9}$ J. Li and S. T. Wu, J. Appl. Phys. 95, 896 (2004). 